\title{
The endothelin receptor antagonist bosentan improves peripheral endothelial function in patients with type 2 diabetes mellitus and microalbuminuria: a randomised trial
}

\author{
A. Rafnsson • F. Böhm • M. Settergren • A. Gonon • \\ K. Brismar • J. Pernow
}

Received: 2 September 2011 / Accepted: 21 November 2011 /Published online: 27 December 2011

(C) Springer-Verlag 2011

\begin{abstract}
Aims/hypothesis Endothelial dysfunction is important in the development of vascular complications in diabetes. Patients with type 2 diabetes have increased production of the vasoconstrictor and pro-inflammatory peptide, endothelin-1. Short-term intra-arterial administration of endothelin antagonists improves endothelium-dependent vasodilatation in patients with type 2 diabetes. We tested the hypothesis that oral administration of the dual endothelin receptor antagonist, bosentan, improves peripheral endothelial function in patients with type 2 diabetes and microalbuminuria.

Methods This placebo-controlled and double-blind study was performed on 46 patients with type 2 diabetes and microalbuminuria (urine albumin/creatinine ratio $>3 \mathrm{mg} / \mathrm{mmol}$ ) at a medical university department. Patients were randomised to bosentan, $125 \mathrm{mg}$ two times per day $(n=28)$, or placebo $(n=28)$ for 4 weeks. The computer-generated randomisation code was kept in sealed envelopes. Patients
\end{abstract}

\footnotetext{
A. Rafnsson · F. Böhm $\cdot$ M. Settergren $\cdot$ A. Gonon $\cdot$ J. Pernow Karolinska Institutet, Department of Medicine, Cardiology Unit, Karolinska University Hospital,

Solna, 17176 Stockholm, Sweden

\section{K. Brismar}

Karolinska Institutet, Department of Molecular Medicine and Surgery, Endocrinology and Diabetology Unit, Karolinska University Hospital,

Stockholm, Sweden
}

\author{
A. Rafnsson $(\bowtie)$ \\ Karolinska Institutet, Department of Cardiology, \\ Karolinska University Hospital, \\ Solna, 17176 Stockholm, Sweden \\ e-mail: arnar.rafnsson@karolinska.se
}

and people doing examinations or assessing outcomes were blinded. The primary endpoint was change in microvascular endothelium-dependent vasodilatation, based on change in digital reactive hyperaemia index. The secondary endpoint was change in brachial artery flow-mediated vasodilatation.

Results Reactive hyperaemia index increased from $1.73 \pm$ 0.43 (mean $\pm \mathrm{SD}$ ) at baseline to $2.08 \pm 0.59$ at follow-up ( $p<$ $0.05)$ in the bosentan group $(n=22)$, but did not change in the placebo group $(1.84 \pm 0.49$ to $1.87 \pm 0.47 ; n=24)$. The change in reactive hyperaemia index from baseline was greater in the bosentan group than in the placebo group $(p<0.05)$. Nitroglycerine-induced digital hyperaemia was not affected. Brachial artery flow-mediated vasodilatation and blood pressure did not change during treatment.

Conclusions/interpretation Oral treatment of 4 weeks duration with the dual endothelin receptor antagonist, bosentan, improves peripheral endothelial function in patients with type 2 diabetes and microalbuminuria.

Trial Registration: ClinicalTrial.gov NCT01357109; Karolinska Clinical Trial Registration Identifier CT20090017 (see www.kctr.se)

Funding: Research Council of Sweden, Swedish Heart and Lung Foundation, Novo Nordisk Foundation, Karolinska Institutet/Stockholm County Council Strategic Cardiovascular Programme, Gustav V and Queen Victoria Foundation, the Family Erling Persson Foundation, Actelion Pharmaceuticals and Actelion Research Award.

Keywords Bosentan · Endo-Pat - Endothelial dysfunction . Endothelin-1 · Endothelin receptor antagonist . FMD . Microalbuminuria $\cdot$ Placebo $\cdot$ Randomised controlled trial . Type 2 diabetes 


$\begin{array}{ll}\text { Abbreviations } \\ \mathrm{EIDV} & \text { Endothelium-independent vasodilatation } \\ \mathrm{ET}_{\mathrm{A}} & \text { Endothelin receptor A } \\ \mathrm{ET}_{\mathrm{B}} & \text { Endothelin receptor B } \\ \mathrm{FMD} & \text { Flow-mediated dilatation } \\ \text { IGFBP-1 } & \text { IGF binding protein-1 } \\ \text { PAT } & \text { Pulse amplitude tonometry } \\ \text { RHI } & \text { Reactive hyperaemia index }\end{array}$

\section{Introduction}

Type 2 diabetes carries a substantially increased risk of macroand microvascular complications, including coronary artery disease, nephropathy, retinopathy and non-healing foot ulcers [1]. The available data suggest that endothelial dysfunction, characterised by reduced bioavailability of nitric oxide, is important in the pathogenesis of diabetic angiopathy due to increased vascular tone, vascular inflammation and oxidative stress $[2,3]$.

Another potentially important factor in endothelial dysfunction in type 2 diabetes is endothelin-1, which exerts potent vasoconstrictor and pro-inflammatory actions via activation of the two receptors, endothelin receptor $\mathrm{A}\left(\mathrm{ET}_{\mathrm{A}}\right)$ and endothelin receptor $\mathrm{B}\left(\mathrm{ET}_{\mathrm{B}}\right)$. Both receptors are present on vascular smooth muscle cells, which mediate vasoconstriction, while the $\mathrm{ET}_{\mathrm{B}}$ is also present on endothelial cells, which mediate vasodilatation. Elevated levels of endothelin-1 are found in patients with type 2 diabetes [4]. We have recently shown that short-term local intra-arterial infusions of endothelin receptor antagonists acutely improved endotheliumdependent vasodilatation in patients with insulin resistance, and in patients with type 2 diabetes and vascular complications [5]. The improvement in endothelium-dependent vasodilatation in insulin-resistant patients was achieved using dual $\mathrm{ET}_{\mathrm{A}} /$ $\mathrm{ET}_{\mathrm{B}}$ blockade, whereas no effect was observed following selective $\mathrm{ET}_{\mathrm{A}}$ blockade, suggesting that blockade of the $\mathrm{ET}_{\mathrm{A}}$ and $\mathrm{ET}_{\mathrm{B}}$ together is important for achieving therapeutic vascular effects [6]. Furthermore, a $60 \mathrm{~min}$ local intra-arterial infusion of an $\mathrm{ET}_{\mathrm{A}}$ antagonist induced a twofold increase in skin capillary flow velocity in patients with type 2 diabetes and microangiopathy, whereas no effect was observed in nondiabetic controls [7]. These observations indicate that shortterm administration of endothelin receptor antagonists acutely improves micro- and macrovascular function, and may be of therapeutic value for patients with glucometabolic perturbations and cardiovascular disease. Data on the effect of longterm oral administration of the above antagonists on endothelial function are limited. A recent study demonstrated that 6 months of treatment with the oral $\mathrm{ET}_{\mathrm{A}}$ antagonist, atrasentan, improved coronary endothelial function in patients with atherosclerosis [8]. However, only two of 25 patients included in the active treatment group of that study had diabetes. Thus, the effect of prolonged oral administration of endothelin receptor antagonists on vascular function in patients with type 2 diabetes and angiopathy remains to be determined. We therefore sought to evaluate the effect of oral administration of the dual $\mathrm{ET}_{\mathrm{A}}$ and $\mathrm{ET}_{\mathrm{B}}$ antagonist, bosentan, on vascular function in patients with type 2 diabetes and microalbuminuria.

\section{Methods}

Patients Patients with type 2 diabetes of at least 2 years duration and microalbuminuria were recruited from the Department of Endocrinology, Metabolism and Diabetes at Karolinska University Hospital from Autumn 2007 to Spring 2010. Patients were classified as having diabetes mellitus if fasting blood glucose exceeded $7.0 \mathrm{mmol} / \mathrm{l}$ on at least two occasions or if the blood glucose concentration was $>11.0 \mathrm{mmol} / 1$ at $2 \mathrm{~h}$ after an oral glucose load $(75 \mathrm{~g})$. Albuminuria was defined as urine albumin concentration $>20 \mu \mathrm{g} / \mathrm{l}$ or $>30 \mu \mathrm{g}$ per $12 \mathrm{~h}$ or an albumin/creatinine ratio of $>3.0 \mathrm{mg} / \mathrm{mmol}$. Diabetic retinopathy was determined after examination by a specialist in ophthalmology with fundus microscopy, and classified as either non-proliferative or proliferative diabetic retinopathy. Neuropathy was assessed by foot examination (autonomic, sensory and motor function) and by symptoms of polyneuropathy. Patients were excluded if they had: (1) had a myocardial infarction or unstable angina within the last 3 months; (2) decompensated heart failure; (3) changed dose of any vasodilator drug during the preceding 6 weeks; (4) childbearing potential; (5) impaired hepatic function (two times the normal limit of serum aminotransferases); (6) ongoing treatment with glibenclamide, ciclosporin or warfarin; or (7) any concomitant disease that might have interfered with the ability to comply with or complete the study protocol. The study protocol was conducted according to the Declaration of Helsinki.

Study design The study was randomised, double-blind and placebo-controlled with two parallel groups. The study protocol was approved by the local Ethics Review Board and the Swedish Medical Product Agency. Informed consent was obtained from all patients. Following baseline investigations of microvascular and macrovascular endothelial function and blood sampling, 56 patients were randomised to treatment with bosentan or placebo. Bosentan treatment was started at a dose of $62.5 \mathrm{mg}$ twice daily for 2 weeks, and in the absence of side effects, the dose was increased to $125 \mathrm{mg}$ twice daily for 2 weeks. After the total treatment period of 4 weeks the patients were re-examined. Treatment compliance was checked by pill count. A computer-generated randomisation list was delivered by the producer of the drug/ placebo (Actelion, Allschwil, Switzerland). The participants were randomised in blocks of four. The randomisation code 
was kept in sealed envelopes, which were not opened until completion of data collection and analyses.

Peripheral endothelial function testing The investigations were performed following a light breakfast in the morning after $20 \mathrm{~min}$ rest and with the patient in a supine position. The patients were asked to refrain from caffeine-containing drinks or tobacco consumption, and intake of drugs was withheld for the 12 preceding hours and until after the examinations. Non-invasive determination of digital endothelial function was measured with a pulse amplitude tonometry (PAT) device placed on the tip of each index finger (EndoPAT2000; Itamar Medical, Caesarea, Israel). The PAT device comprises a pneumatic plethysmograph that applies a uniform pressure to the surface of the distal finger, allowing measurement of pulse volume changes. The inflation pressure of the digital device was electronically set to $10 \mathrm{mmHg}$ below diastolic blood pressure or $70 \mathrm{mmHg}$ (whichever was lower). The PAT signal was recorded at baseline and following $5 \mathrm{~min}$ arterial occlusion using an inflatable cuff, while the contralateral arm served as a control. The blood pressure cuff was inflated to $60 \mathrm{mmHg}$ higher than the systolic pressure or at least $200 \mathrm{mmHg}$ for $5 \mathrm{~min}$. Lack of residual pulsatility was monitored throughout the occlusion period. The post-occlusive hyperaemia stimulates endothelium-dependent vasodilatation, causing an increase in digital pulse amplitude [9, 10]. Pulse amplitude was recorded electronically in both fingers and analysed by a computerised, automated algorithm (Itamar Medical). The change from the baseline measurement is expressed as the reactive hyperaemia index (RHI), which in part reflects vasodilator function of the digital microcirculation [11]. Previous evaluation of this method has demonstrated that RHI is to a large part dependent on nitric oxide bioavailability [9]. Endothelium-independent vasodilatation (EIDV) was determined following sublingual administration of nitroglycerine $(0.4 \mathrm{mg})$. The ratio between the maximal pulse amplitude following nitroglycerine administration and baseline pulse amplitude was used to calculate EIDV. The coefficient of variation for the Endo-PAT device was $12 \%$ in our lab.

Flow-mediated vasodilatation of the brachial artery Macrovascular endothelial function was determined by analysis of flow-mediated dilatation (FMD) of the brachial artery during reactive hyperaemia following forearm ischaemia as previously described in detail [5]. An $8 \mathrm{MHz}$ linear-array transducer connected to a device (Acuson Sequoia; Acuson, Mountain View, CA, USA) was used in parallel with the determination of digital PAT. Baseline images were saved every third second for $1 \mathrm{~min}$ and a mean value was calculated from these values. The artery was continuously imaged for 3 min during the hyperaemia following release of the pressure cuff, in order to determine endothelium-dependent vasodilatation. A mean value was calculated from three recordings at maximum dilatation. EIDV was determined following sublingual administration of nitroglycerine. All images were analysed using proprietary software (Brachial Analyser; Medical Imaging Applications, Iowa City, IA, USA) by a technician blinded to treatment allocation. The coefficient of variation for FMD was $19 \%$ in our lab.

Laboratory investigations Fasting plasma glucose, $\mathrm{HbA}_{1 \mathrm{c}}$, blood lipids and liver function tests were done by standard methods according to local laboratory routines. IGF-1 was analysed by RIA and calculated using an age-adjusted SD score: $(10 \log [$ IGF - I level $]-0.00625 \times$ age -2.555$) /$ 0.104 [12]. IGF binding protein-1 (IGFBP-1) was determined by RIA [13]. Endothelin-1 was measured using ELISA (R\&D Systems, Minneapolis, MN, USA). High-sensitivity C-reactive protein was analysed using turbidimetry (Beckman Coulter, Fullerton, CA, USA).

Statistical analysis Data are presented as mean and SD. A two-sided value of $p<0.05$ was considered significant. The data for analyses between the groups were calculated as change from baseline after completed therapy. Group comparisons were made using a $t$ test. Data that were not normally distributed were log-transformed. The primary endpoint of the study was change in RHI. The secondary endpoint was change in brachial artery FMD. The number of patients per group needed to detect a difference in RHI of 0.3 with a power of $80 \%$ and with a two-tailed $t$ test at the $5 \%$ level was calculated to be 22, with SD of 0.35 for difference in RHI. The RHI improvement by 0.3 was based on a previous study [10].

\section{Results}

Characteristics of study participants In the bosentan group, three patients stopped the study because of adverse events (one due to facial flushing and oedema, two due to respiratory tract infection) and three patients were excluded due to protocol violation (one patient due to only $4 \mathrm{~min}$ arterial occlusion, two patients due to change in vasodilatory medication during the study period). In the placebo group, four patients were excluded. One of these suffered an ischaemic stroke, two patients did not take the study medication as instructed and one patient was excluded due to a concomitant condition that interfered with his/her ability to comply with the study protocol. A total of 46 patients remained in the final analysis (Fig. 1). The two groups were well matched at baseline with no significant differences in their baseline characteristics or medication (Table 1).

Digital endothelial function The primary endpoint was change in digital RHI from baseline to follow-up as measured 
Fig. 1 Flow chart of the study

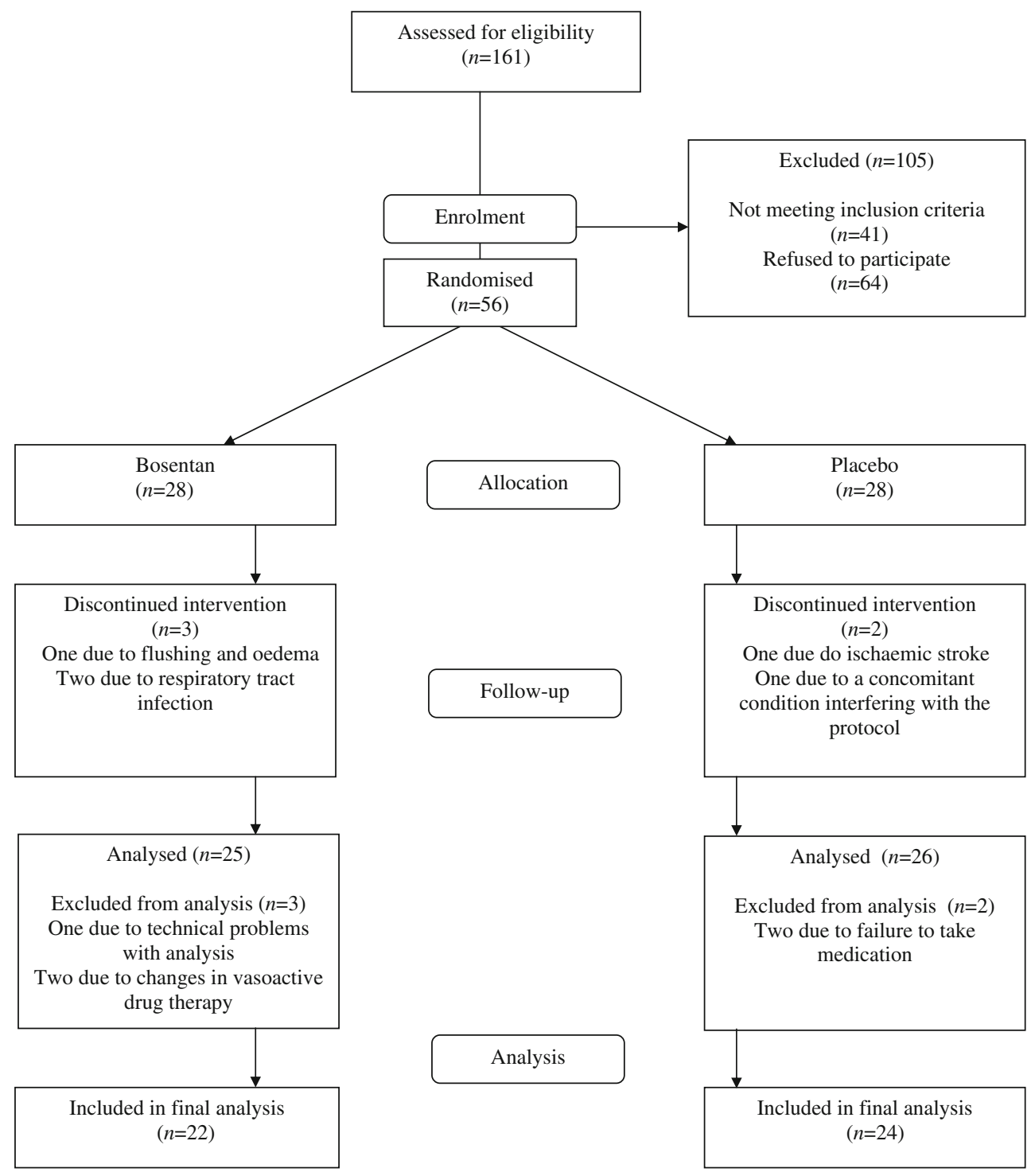

by the Endo-PAT device. Baseline RHI was $1.82 \pm 0.50$ (mean $\pm \mathrm{SD}$ ) for the entire study population. Baseline RHI was similar in the two groups. RHI did not change significantly from baseline $(1.84 \pm 0.49)$ to follow-up $(1.87 \pm 0.47)$ in the placebo group. In the bosentan-treated group, RHI increased from $1.73 \pm 0.43$ at baseline to $2.08 \pm$ 0.59 at follow-up $(p<0.05)$ (Fig. 2a). The change in RHI during the 4 weeks of treatment with bosentan was significantly greater than that in the placebo group $(0.38,95 \% \mathrm{CI}$ $0.02,0.74)$ (Fig. 2b). The change in RHI did not correlate with change in systolic blood pressure $(r=0.16 ; p=0.28)$. The nitroglycerine-induced PAT signal was not affected by placebo or bosentan treatment $(1.20 \pm 0.44$ at baseline, $1.24 \pm 0.30$ at follow-up).

Flow-mediated vasodilatation of the brachial artery Baseline brachial artery FMD was comparable in the two groups. After 4 weeks of treatment there was no significant change in FMD
$(0.016,95 \%$ CI $-1.38,1.0)$ (Table 2) or nitroglycerineinduced brachial artery dilatation in either group.

Biochemical analyses Treatment with bosentan resulted in a significant drop in haemoglobin from 134 to $127 \mathrm{~g} / 1$ ( $p<$ 0.001 ) (Table 2). There was no significant change in urine albumin/creatinine ratio in either group. During treatment there were no changes in lipid profile or blood glucose levels. Plasma levels of endothelin-1 increased from $1.5 \pm$ $0.6 \mathrm{pg} / \mathrm{ml}($ mean $\pm \mathrm{SD})$ at baseline to $2.6 \pm 1.2 \mathrm{pg} / \mathrm{ml}(p<$ 0.001 ) in the bosentan group, an increase that could be expected due to the reduced $\mathrm{ET}_{\mathrm{B}}$-mediated clearance of endothelin-1. Endothelin-1 levels did not change in the placebo group $(1.8 \pm 0.9$ vs $1.7 \pm 0.7 \mathrm{pg} / \mathrm{ml}$, baseline vs follow-up). IGF-1 and IGFBP-1 did not differ at baseline between groups and were not affected by treatment. There was no change in hepatic transaminases during the treatment period. 
Table 1 Baseline characteristics

\begin{tabular}{|c|c|c|}
\hline Characteristic & Placebo group & $\begin{array}{l}\text { Bosentan } \\
\text { group }\end{array}$ \\
\hline$n$ & 24 & 22 \\
\hline Age (years) & $63 \pm 9$ & $62 \pm 8$ \\
\hline Men $(n)$ & 19 & 18 \\
\hline Women $(n)$ & 5 & 4 \\
\hline $\operatorname{BMI}\left(\mathrm{kg} / \mathrm{m}^{2}\right)$ & $31.5 \pm 4.0$ & $28.9 \pm 7.4$ \\
\hline Waist circumference $(\mathrm{cm})$ & $111 \pm 11$ & $109 \pm 10$ \\
\hline Current smokers $(n)$ & 1 & 2 \\
\hline Diabetes duration (years) & $17.7 \pm 9.6$ & $14.9 \pm 6.3$ \\
\hline Coronary artery disease $(n)$ & 9 & 10 \\
\hline Peripheral artery disease $(n)$ & 4 & 0 \\
\hline \multicolumn{3}{|l|}{ Retinopathy } \\
\hline Non-proliferative $(n)$ & 10 & 8 \\
\hline Proliferative $(n)$ & 5 & 2 \\
\hline \multicolumn{3}{|l|}{ Neuropathy } \\
\hline Moderate $(n)$ & 5 & 7 \\
\hline Severe $(n)$ & 6 & 2 \\
\hline \multicolumn{3}{|l|}{ Treatments } \\
\hline Insulin $(n)$ & 16 & 18 \\
\hline Oral glucose-lowering agents $(n)$ & 16 & 13 \\
\hline Aspirin $(n)$ & 15 & 13 \\
\hline Beta-blocker $(n)$ & 8 & 12 \\
\hline $\mathrm{ACE}$ inhibitor/ARB $(n)$ & 19 & 20 \\
\hline Calcium channel blocker $(n)$ & 9 & 9 \\
\hline Statins $(n)$ & 13 & 14 \\
\hline
\end{tabular}

Values are mean \pm SD unless otherwise indicated

$\mathrm{ARB}$, angiotensin receptor blocker

\section{Discussion}

Endothelial dysfunction, characterised as impaired bioavailability of nitric oxide, is considered to be important in the early pathogenesis of vascular complications associated with diabetes $[2,3]$. This study demonstrates that 4 weeks of oral treatment with the dual endothelin receptor antagonist, bosentan, improved digital RHI in patients with type 2 diabetes and albuminuria, suggesting that dual endothelin receptor blockade improves peripheral endothelial function in patients with type 2 diabetes and vascular complications.

Several observations indicate that endothelin-1 may be involved in the pathogenesis of vascular complications in type 2 diabetes. Elevated plasma levels of endothelin-1 are found in patients with type 2 diabetes [4]. Short-term local intra-arterial administration of endothelin receptor antagonists improved endothelium-dependent vasodilatation in participants with insulin resistance [6], and in patients with type 2 diabetes mellitus with and without coronary artery
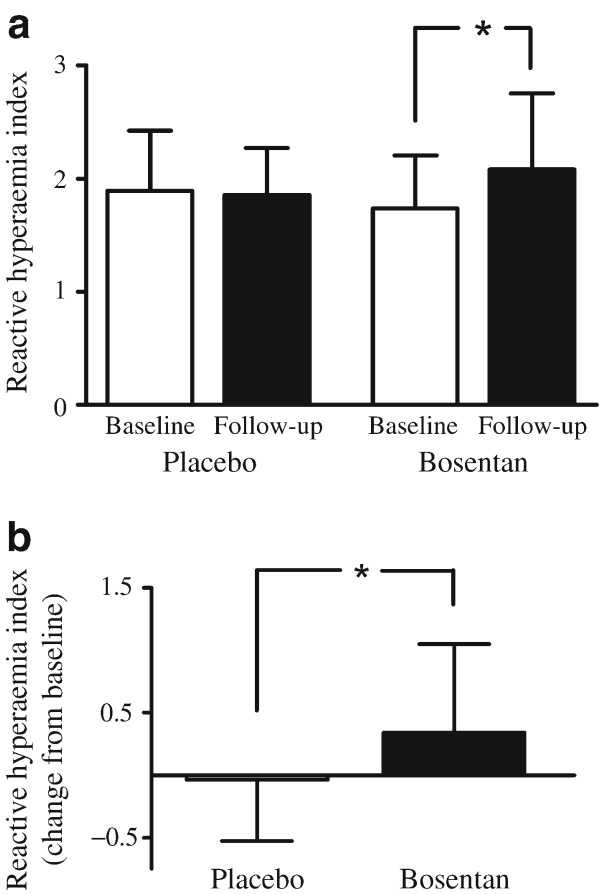

Fig. 2 a Reactive hyperaemia index (RHI) at baseline and follow-up for the placebo and bosentan groups. b Change in RHI from baseline in the two groups. Values are mean $\pm \mathrm{SD} ;{ }^{*} p<0.05$

disease $[5,14]$. Furthermore, intra-arterial endothelin receptor blockade increased digital cutaneous microvascular flow velocity in patients with type 2 diabetes mellitus and microangiopathy [7]. All these previous studies were limited to shortterm intra-arterial administration of endothelin receptor antagonists. In the present study, we investigated the effect of oral administration of a clinically relevant dose of the endothelin receptor antagonist, bosentan, in patients with type 2 diabetes and vascular complications. Bosentan is currently approved for treatment of pulmonary arterial hypertension and digital ulcers in scleroderma. The present results demonstrate that bosentan treatment for 4 weeks significantly increased the digital PAT signal compared with baseline and with placebo. Previous studies have established that a large part of the digital PAT signal during reactive hyperaemia is dependent on microvascular nitric oxide bioavailability [9, 11]. In the present study, nitroglycerine-induced hyperaemia was not affected by bosentan treatment, indicating that endothelium-independent dilatation was not improved. Therefore, our observations suggest that bosentan improved microvascular endothelium-dependent vasodilatation. This is the first study to demonstrate beneficial effects of oral endothelin receptor blockade on peripheral endothelial function in patients with type 2 diabetes mellitus.

Bosentan improved peripheral endothelium-dependent vasodilatation determined as changes in the PAT signal, whereas no change was observed in brachial artery FMD. Previous observations suggest that the PAT signal and FMD 
Table 2 Treatment effects

Values are mean $\pm \mathrm{SD}$

$* * * p<0.001$ vs baseline

VTI, velocity time integral; IGF-1 SD-score, age adjusted IGF-1; ASAT, aspartate aminotransferase; ALAT, alanine aminotransferase; uACR, urine albumin/ creatinine ratio; hsCRP, high-sensitivity C-reactive protein

\begin{tabular}{|c|c|c|c|c|}
\hline \multirow[t]{2}{*}{ Effect } & \multicolumn{2}{|l|}{ Placebo } & \multicolumn{2}{|l|}{ Bosentan } \\
\hline & Baseline & Follow-up & Baseline & Follow-up \\
\hline FMD change $(\%)$ & $3.3 \pm 2.7$ & $3.2 \pm 2.0$ & $3.1 \pm 2.8$ & $3.2 \pm 2.7$ \\
\hline \multicolumn{5}{|l|}{ Brachial artery basal } \\
\hline Diameter (mm) & $3.9 \pm 0.7$ & $3.9 \pm 0.7$ & $3.9 \pm 0.6$ & $4.0 \pm 0.7$ \\
\hline Brachial artery VTI $(\mathrm{cm})$ & $21 \pm 6.9$ & $23 \pm 6.9$ & $29 \pm 11$ & $28 \pm 6.7$ \\
\hline Systolic BP (mmHg) & $151 \pm 25$ & $150 \pm 21$ & $149 \pm 24$ & $143 \pm 18$ \\
\hline Diastolic BP (mmHg) & $78 \pm 9$ & $79 \pm 8$ & $81 \pm 10$ & $77 \pm 11$ \\
\hline Haemoglobin (g/l) & $136.5 \pm 13.6$ & $137.2 \pm 15.1$ & $134.0 \pm 16.1$ & $127.3 \pm 15.1 * * *$ \\
\hline Glucose (mmol/l) & $9.5 \pm 3.9$ & $9.9 \pm 3.6$ & $8.6 \pm 2.5$ & $8.4 \pm 2.7$ \\
\hline Insulin (pmol/1) & $107.5 \pm 143.1$ & $95.8 \pm 97.6$ & $129.2 \pm 121.3$ & $118.3 \pm 93.1$ \\
\hline IGF-1 (SD score) & $-0.47 \pm 1.4$ & $-0.37 \pm 1.6$ & $-0.39 \pm 1.2$ & $-0.87 \pm 1.1$ \\
\hline IGFBP-1 ( $\mu \mathrm{g} / \mathrm{l})$ & $50.9 \pm 33.8$ & $51.3 \pm 33.8$ & $50.7 \pm 35.6$ & $54.1 \pm 30.7$ \\
\hline ASAT $(\mu \mathrm{kat} / \mathrm{l})$ & $0.51 \pm 0.45$ & $0.48 \pm 0.25$ & $0.39 \pm 0.14$ & $0.48 \pm 0.31$ \\
\hline ALAT $(\mu \mathrm{kat} / \mathrm{l})$ & $0.61 \pm 0.71$ & $0.56 \pm 0.53$ & $0.47 \pm 0.24$ & $0.58 \pm 0.42$ \\
\hline $\mathrm{uACR}(\mathrm{mg} / \mathrm{mmol})$ & $46.3 \pm 58.0$ & $39.0 \pm 70.7$ & $46.9 \pm 77.1$ & $31.6 \pm 47.0$ \\
\hline Triacylglycerol (mmol/l) & $1.6 \pm 0.8$ & $1.7 \pm 0.7$ & $1.5 \pm 0.7$ & $1.6 \pm 0.7$ \\
\hline Total cholesterol (mmol/l) & $4.0 \pm 0.9$ & $4.1 \pm 1.0$ & $3.7 \pm 0.7$ & $3.7 \pm 0.5$ \\
\hline HDL-cholesterol (mmol/l) & $1.0 \pm 0.3$ & $1.0 \pm 0.3$ & $0.9 \pm 0.1$ & $0.8 \pm 0.2$ \\
\hline LDL-cholesterol (mmol/l) & $2.4 \pm 0.9$ & $2.4 \pm 0.9$ & $2.1 \pm 0.5$ & $2.1 \pm 0.5$ \\
\hline $\mathrm{HbA}_{1 \mathrm{c}}(\%)$ & $8.0 \pm 1.4$ & $8.0 \pm 1.4$ & $7.4 \pm 1.1$ & $7.4 \pm 1.0$ \\
\hline $\mathrm{HbA}_{1 \mathrm{c}}(\mathrm{mmol} / \mathrm{mol})$ & $64.0 \pm 15.0$ & $64.3 \pm 15.4$ & $57.8 \pm 12.1$ & $56.9 \pm 11.1$ \\
\hline Creatinine $(\mu \mathrm{mol} / \mathrm{l})$ & $101.9 \pm 51.3$ & $107.3 \pm 56.6$ & $125.2 \pm 96.0$ & $115.7 \pm 85.0$ \\
\hline hsCRP (mg/l) & $3.0 \pm 3.3$ & $3.4 \pm 2.9$ & $3.2 \pm 3.2$ & $4.8 \pm 7.7$ \\
\hline Endothelin-1 (pg/ml) & $1.8 \pm 0.9$ & $1.7 \pm 0.8$ & $1.5 \pm 0.6$ & $2.6 \pm 1.1 * * *$ \\
\hline
\end{tabular}

provide distinct information on vascular function in small vessels versus conduit vessels [15]. Since the PAT signal reflects changes in smaller vessels, including the microvasculature in the fingertip [11], the present findings may indicate that bosentan improves endothelial function in small vessels rather than in large conduit arteries (brachial artery). This is of particular relevance in type 2 diabetes, which is characterised by early disturbance of microvascular endothelial function [16]. In fact, the available data suggest that microvascular endothelial dysfunction is associated with increased cardiovascular risk and high prevalence of angiopathic complications in type 2 diabetes [2]. Therefore, the improvement of microvascular endothelial function could be important in the treatment of patients with type 2 diabetes. The use of the EndoPAT device to determine endothelial function has been validated as a method in several studies, and endothelial dysfunction determined by the EndoPAT has been shown to predict cardiovascular events $[17,18]$.

Although the mechanism behind the positive effect of bosentan on endothelial function that was observed in the present study is beyond the study's primary purpose, some of the potential mechanisms are worthy of comment. It has previously been demonstrated that exogenous endothelin-1 induces endothelial dysfunction [19] and endothelin receptor antagonists improve endothelial function $[7,14,19]$ in healthy humans and in patients with atherosclerosis and type 2 diabetes, acting via a mechanism related to increased bioavailability of nitric oxide. Endothelin-1 may inhibit the production of nitric oxide via attenuation of nitric oxide synthase production and activity [20]. Another potential mechanism is via a decrease in reactive oxygen species, which reduce the bioavailability of nitric oxide. Endothelin-1 stimulates the production of reactive oxygen species [21], which are known to be an important feature of endothelial dysfunction in patients with type 2 diabetes [22, 23].

In our study, there was a non-significant reduction in the urine albumin/creatinine ratio. Previous experimental and clinical studies have demonstrated beneficial effects of endothelin receptor blockade on proteinuria [24-26]. The non-selective endothelin receptor antagonist, avosentan, reduced albuminuria when added to standard treatment in patients with type 2 diabetes and overt nephropathy [26]. However, that study was prematurely stopped due to significant fluid overload and congestive heart failure. Furthermore, Kohan et al. recently demonstrated a reduction of the urine 
albumin/creatinine ratio in patients with type 2 diabetes and nephropathy who were given the $\mathrm{ET}_{\mathrm{A}}$ selective antagonist, atrasentan [25]. In the present study, we also observed a nonsignificant trend towards reduced blood pressure in the bosentan group ( -6 and $-4 \mathrm{mmHg}$ in systolic and diastolic blood pressure, respectively). This is of the same magnitude as that found in the studies using avosentan and atrasentan in patients with type 2 diabetes $[25,26]$. It cannot be ruled out that the blood pressure response may have contributed to the change in the PAT signal following bosentan treatment. However, lower blood pressure was associated with an impaired PAT signal [15], suggesting that, if anything, a reduction in blood pressure would underestimate the PAT response.

Study limitations The study was performed on a limited number of patients. The sample size was calculated for an expected change in RHI of 0.3 units [10], and this difference was reached in the bosentan-treated group. However, the changes in brachial artery FMD were smaller and did not reach significant difference. The intra-individual variation was slightly higher with FMD than for Endo-PAT, which, at least in part, can explain the difference in the results using these two methods. The duration of treatment was 4 weeks, and it is possible that a longer period of treatment is required for effects on vascular remodelling to occur. It cannot be ruled out that positive effects would have been observed in conduit artery FMD after a longer treatment duration. Another limitation is that we did not include a control group given a blood pressure-lowering drug devoid of endothelial effects. However, it is difficult to identify a blood pressure-lowering drug that does not directly or indirectly affect endothelial function. Previous studies using local intra-arterial administration of endothelin receptor antagonists have demonstrated improvements in endothelial function without affecting blood pressure $[5,6]$, suggesting that the effect may be independent of any change in systemic blood pressure. However, the exact mechanism by which bosentan improves vascular function remains to be established in future studies.

Conclusions The present study demonstrates that the dual endothelin receptor antagonist, bosentan, improves peripheral endothelial function in patients with type 2 diabetes and microalbuminuria. Although the observations need to be reproduced in larger randomised studies, the data suggest that endothelin receptor blockade may represent an important novel therapeutic strategy for patients with vascular complications due to type 2 diabetes.

Acknowledgements We would like to thank A. Lindström, M. Wallin and M. Lidin (Karolinska Institute, Department of Medicine, Cardiology Unit) for their invaluable work and skilful technical assistance.
Funding This work was supported by grants from the Research Council of Sweden, the Swedish Heart and Lung Foundation, Novo Nordisk Foundation, Karolinska Institutet/Stockholm County Council Strategic Cardiovascular Programme, Gustav V and Queen Victoria Foundation, the Family Erling Persson Foundation, Actelion Pharmaceuticals and Actelion Research Award.

Duality of interest The authors declare that there is no duality of interest associated with this manuscript. Actelion took no part in the design of the study, data collection or the writing of the manuscript.

Contribution statement All authors took part in the conception and design of the study, as well as either drafting or critically revising the manuscript. All authors have approved the final version of the manuscript.

\section{References}

1. Gall MA, Rossing P, Skott P et al (1991) Prevalence of micro- and macroalbuminuria, arterial hypertension, retinopathy and large vessel disease in European type 2 (non-insulin-dependent) diabetic patients. Diabetologia 34:655-661

2. Tooke JE, Hannemann MM (2000) Adverse endothelial function and the insulin resistance syndrome. J Intern Med 247:425-431

3. Creager MA, Luscher TF, Cosentino F, Beckman JA (2003) Diabetes and vascular disease: pathophysiology, clinical consequences, and medical therapy: part I. Circulation 108:1527-1532

4. Takahashi K, Ghatei MA, Lam HC, O'Halloran DJ, Bloom SR (1990) Elevated plasma endothelin in patients with diabetes mellitus. Diabetologia 33:306-310

5. Settergren M, Bohm F, Ryden L, Pernow J (2008) Cholesterol lowering is more important than pleiotropic effects of statins for endothelial function in patients with dysglycaemia and coronary artery disease. Eur Heart J 29:1753-1760

6. Shemyakin A, Bohm F, Wagner H, Efendic S, Bavenholm P, Pernow J (2006) Enhanced endothelium-dependent vasodilatation by dual endothelin receptor blockade in individuals with insulin resistance. J Cardiovasc Pharmacol 47:385-390

7. Settergren M, Pernow J, Brismar K, Jorneskog G, Kalani M (2008) Endothelin-A receptor blockade increases nutritive skin capillary circulation in patients with type 2 diabetes and microangiopathy. $\mathrm{J}$ Vasc Res 45:295-302

8. Reriani M, Raichlin E, Prasad A et al (2010) Long-term administration of endothelin receptor antagonist improves coronary endothelial function in patients with early atherosclerosis. Circulation 122:958-966

9. Nohria A, Gerhard-Herman M, Creager MA, Hurley S, Mitra D, Ganz P (2006) Role of nitric oxide in the regulation of digital pulse volume amplitude in humans. J Appl Physiol 101:545-548

10. Bonetti PO, Barsness GW, Keelan PC et al (2003) Enhanced external counterpulsation improves endothelial function in patients with symptomatic coronary artery disease. J Am Coll Cardiol 41:1761-1768

11. Hamburg NM, Benjamin EJ (2009) Assessment of endothelial function using digital pulse amplitude tonometry. Trends Cardiovasc Med 19:6-11

12. Hilding A, Brismar K, Degerblad M, Thoren M, Hall K (1995) Altered relation between circulating levels of insulin-like growth factor-binding protein-1 and insulin in growth hormone-deficient patients and insulin-dependent diabetic patients compared to that in healthy subjects. J Clin Endocrinol Metab 80:2646-2652 
13. Povoa G, Roovete A, Hall K (1984) Cross-reaction of serum somatomedin-binding protein in a radioimmunoassay developed for somatomedin-binding protein isolated from human amniotic fluid. Acta Endocrinol (Copenh) 107:563-570

14. Mather KJ, Mirzamohammadi B, Lteif A, Steinberg HO, Baron AD (2002) Endothelin contributes to basal vascular tone and endothelial dysfunction in human obesity and type 2 diabetes. Diabetes 51:3517-3523

15. Hamburg NM, Palmisano J, Larson MG et al (2011) Relation of brachial and digital measures of vascular function in the community: the Framingham Heart Study. Hypertension 57:390-396

16. Tooke JE (2000) Possible pathophysiological mechanisms for diabetic angiopathy in type 2 diabetes. J Diabetes Complications 14:197-200

17. Rubinshtein R, Kuvin JT, Soffler M et al (2010) Assessment of endothelial function by non-invasive peripheral arterial tonometry predicts late cardiovascular adverse events. Eur Heart J 31: $142-1148$

18. Hamburg NM, Keyes MJ, Larson MG et al (2008) Cross-sectional relations of digital vascular function to cardiovascular risk factors in the Framingham Heart Study. Circulation 117:2467-2474

19. Böhm F, Ahlborg G, Pernow J (2002) Endothelin-1 inhibits endothelium-dependent vasodilatation in the human forearm: reversal by ETA receptor blockade in patients with atherosclerosis. Clin Sci (Lond) 102:321-327

20. Gonon AT, Erbas D, Broijersen A, Valen G, Pernow J (2004) Nitric oxide mediates protective effect of endothelin receptor antagonism during myocardial ischemia and reperfusion. Am J Physiol Heart Circ Physiol 286:H1767-H1774

21. Wedgwood S, Dettman RW, Black SM (2001) ET-1 stimulates pulmonary arterial smooth muscle cell proliferation via induction of reactive oxygen species. Am J Physiol Lung Cell Mol Physiol 281:L1058-L1067

22. Rask-Madsen C, King GL (2007) Mechanisms of disease: endothelial dysfunction in insulin resistance and diabetes. Nat Clin Pract Endocrinol Metab 3:46-56

23. Jansson PA (2007) Endothelial dysfunction in insulin resistance and type 2 diabetes. J Intern Med 262:173-183

24. Barton M (2010) Therapeutic potential of endothelin receptor antagonists for chronic proteinuric renal disease in humans. Biochim Biophys Acta 1802:1203-1213

25. Kohan DE, Pritchett Y, Molitch M et al (2011) Addition of atrasentan to renin-angiotensin system blockade reduces albuminuria in diabetic nephropathy. J Am Soc Nephrol 22:763-772

26. Mann JF, Green D, Jamerson K et al (2010) Avosentan for overt diabetic nephropathy. J Am Soc Nephrol 21:527-535 